\title{
A new approach for directing proactive sewer maintenance
}

\section{R. A. Fenner, L. Sweeting and M. J. Marriott}

\section{Pizey, South West Water Ltd}

I would like to offer the following comments on the authors' paper.

(a) I note nine water companies were contacted-was South West Water one of these? If so, I would be interested to know who was your contact at SWW. If not, I would be happy to act as point of contact for any future studies.

(b) I think your assessment of data availability across water companies is most accurate.

(c) I welcome the move away from concentrating on critical sewers to considering the whole network. The bulk of our sewerage customer complaints are associated with noncritical sewers.

(d) Perhaps criticality could be used as a factor in your costing matrix.

(e) I would be interested to know if you think the size of grid squares used greatly affects the results (especially in view of your comment 'each grid square is likely to contain assets of a similar type'). If so, why were $500 \mathrm{~m}$ squares chosen?

(f) Presumably the number of customers affected is more pertinent than the number of customers in a particular grid square. Number of customers upstream would also give an idea of load. Perhaps number of customers per grid square was used as a surrogate for number of customers upstream, as the latter data did not exist?

(g) I am very interested in your conclusion: 'longer foul sewers of small diameter (150 $\mathrm{mm}$ or less) laid at a shallow depth $(0-10 \mathrm{~m})$ and slack gradient' are more susceptible to problems. Presumably with an area-based approach like this, longer sewers are likely to seem to cause more problems. Are you saying that, for two otherwise identical grid squares, each with say $1000 \mathrm{~m}$ of sewer, one with $20 \times 50 \mathrm{~m}$ lengths and one with $10 \times 100 \mathrm{~m}$ lengths, the latter square is likely to have more failure incidents?

\section{Authors' Reply \\ Points (a) to (c)}

Although the correspondent's company was not one of those contributing data to the study, the authors welcome his offer of involvement in any future studies, and are pleased to hear his endorsement of particular conclusions given in the paper.

Point (d) (use of criticality in the costing matrix)

The intention of the methodology was to break away from selecting pipes on the basis of whether they were defined as critical, in order not to skew any proactive work towards such a predetermined subset. Nevertheless, it is true that critical sewers will often be the most expensive to repair (by definition) and on this basis are likely to dominate any cost analysis.

Perhaps the suggestion is to simply rank the grid squares in descending order of criticality, and then to consider the associated costs. This was not recommended as the likelihood and consequence scores which define criticality were intentionally kept separate, in order to better inform the engineering decisions about what, if any, proactive maintenance action should be taken. Criticality on its own masks these components. By using a three-dimensional radar plot it is easy to see the reason for criticality (i.e. whether consequence or likelihood factors dominate), and so it is a straightforward procedure to identify those squares which are relatively cheap to preemptively repair.

Information was not available to enable the costs of repair for each length of pipe in a grid square to be calculated individually, therefore pipes were divided into cost groupings based on pipe size and depth using water company cost factors. From this information, costs were seen to increase significantly above $400 \mathrm{~mm}$ and again above $1 \mathrm{~m}$ diameter, as well as for depths greater than $2.5 \mathrm{~m}$. It can be conceded that deeper (>3 m), larger diameter ( $>900 \mathrm{~mm}$ ) sewers may be classified as critical using the Sewerage Rehabilitation Manual ${ }^{2}$ (SRM) definition, because they will be more costly to repair. While priorities for rehabilitation are set by individual companies, the concept in the proposed methodology for proactive maintenance is to choose those areas which contain pipes which will be the cheapest to repair but which can be shown to have the highest potential risk of future failure. In this regard using criticality as a cost factor may be misleading.

\section{Point (e) size of grid squares}

The use of grid squares was adopted in the methodology to overcome the widespread lack of connectivity between the separate databases containing asset information and event history. A convenient grid square resolution of $500 \times 500 \mathrm{~m}$ was pragmatically chosen for the analysis reported in the paper, because it was easily divisible into the OS $1 \mathrm{~km}$ grid system, and reflected the $500 \times 500 \mathrm{~m}$ squares used on standard 1:1250 scale sewer maps. Using a fractional measure of OS squares aids in the ease of interpretation for site use and for 
background mapping. However, the choice of grid square resolution is intended to be flexible and can be defined within the GIS software to reflect the sewer density in any given drainage area. Thus in rural areas it may not be useful to choose a resolution which produces many empty squares and so a larger grid size may be adopted, at the discretion of the user. A sensitivity analysis to determine the effect of grid size on the results was beyond the scope and resources of the study, but the use of larger squares in urban areas may prevent adequate discrimination in identifying 'hotspots' of sewer failure. Conversely, grid square boundaries may dissect an area with a cluster of higher than average number of failures. With this in mind, the impact of failure in adjacent $500 \times 500 \mathrm{~m}$ squares was considered and found to be undiagnostic, suggesting a $500 \mathrm{~m}$ resolution to be acceptable.

With regard to grid squares containing assets of similar type, this assumes that pipes in relatively close proximity will often be of a similar age, material, and have been subjected to similar construction techniques. Also some of the external factors such as groundwater regime and soil type may be similar, affecting groups of pipes in a similar way. It is, however, possible that traffic loading may be quite variable within a grid square, with heavy vehicles being restricted to the main urban routes, and some squares may contain mixed development, especially if they have been subject to recent property infilling. Nevertheless, it appears attractive to attempt to bring all the pipes in an area up to an acceptable standard as part of a systematic and carefully focused rehabilitation strategy, rather than attempting to target individual sewer lengths, and risk frequent return visits to the same location to tackle similar problems.

\section{Point (f) (significance of number of customers per grid square)} The analysis uses 'number of properties per grid square' in determining the subjectively formulated consequence factor. This is intended to reflect local impacts which affect the customer directly through loss of toilet use, odour and internal and external flooding. (Global impacts such as traffic disruption and environmental pollution which affect the whole community are reflected simply by using road length and past pollution incidents in a square.)

If an event occurs once and is dealt with efficiently, conveniently and successfully this may actually enhance the customer's view of the company. However, if the event recurs or is not dealt with professionally, complaints will arise. The objective of the local factor in the consequence algorithm (Events/no properties + repeat events/total events) is to highlight those areas where the tolerance of customers has been lowered by previous events. Similarly, areas where many events have been reported in the neighbourhood will lead to a negative view of the water utility by the whole community.

Using the number of events per property takes into account the density of population in each grid square. Thus ten events in a square with three houses will indicate far worse performance than ten events in a square with 600 houses. The other measure is the number of repeat events occurring affecting the same customers, therefore the fraction of all events which are repeat events (affecting the same property address) is incorporated in the consequence algorithm. Importantly, all these measures are capable of being calculated automatically within the GIS software.

Of course the algorithm can be refined and the authors have developed an advanced consequence based on a series of weighting factors to reflect the type of failure experienced (e.g. total loss of service versus internal foul flooding versus stormwater garden flooding, etc.). This has the advantage of allowing the weights to be adjusted to reflect current customer and company priorities. Applying both algorithms to data from one drainage area in the north of England produced the same critical grid squares, suggesting the analysis was not very sensitive to the calculation of consequence.

The discusser suggests taking into account the number of customers upstream, but this would be difficult to calculate from the structure of the databases from which the authors were working. Moreover, this may be a more appropriate factor to consider in water distribution networks, where bursts of a water main may cut off supply to all customers beyond the point of failure. Serviceability failures on sewer pipes tend to have more local impacts in terms of flooding properties close to the point of failure, with upstream customers often unaffected. Identifying accurate numbers of customers tangibly affected by any event was not possible from the data available.

\section{Point (g) (more failure on longer sewers)}

The conclusion concerning longer manhole-to-manhole lengths of sewer being more prone to failure was based on the results of the analysis of individual pipe lengths, using the Bayesian statistical model. The majority of event data studied related to serviceability problems such as blockage (approximately 90\% of the recorded failures), with most water companies at the time not keeping a separate collapse database. Although some of the blockage problems were likely to have arisen from unknown collapses, failure in this context is far more likely to mean soft blockages rather than structural collapse.

Three pipe length categories were defined and the longest length (between $50 \mathrm{~m}$ and $200 \mathrm{~m}$ ) was most clearly associated with these past serviceability failures. We can speculate on the reason for this, such as the greater difficulty of rodding and clearing blockages as the distance between manholes increases. Also longer lengths may give rise to the probability of finding an increased number of misaligned or displaced pipe joints. The reason pipe length was studied as a potential diagnostic factor was to use this as a crude indicator of the number of lateral connections (assuming rough uniformity of pipe connections per metre). Thus longer lengths of pipe may suffer from a greater number of protruding or poorly made connections, which may act as sites for material to accumulate and cause blockage.

In the case of the grid squares analysis it was recognised that the total length of pipe is variable for each individual grid square, and although pipe length may be important in prediction, it was also felt important to look at the variables independent of pipe length to identify characteristics associated with failure. So, for example, we can ask whether it is the material of the pipe (e.g. clay) that is contributing to greater than average failure in a square, or is it the higher than average length of pipe in the square. Thus a homogeneous data set was 
created which expressed the length of pipe associated with each physical variable as a percentage of the total pipe length in each square. The overall mean percentages of pipes with a particular characteristic (e.g. diameter, material, depth, etc.) in the squares which had a failure history were compared with those squares which had no failure history. In searching for possible causal factors associated with those grid squares which had a previous event history, this eliminated the problem of squares with longer lengths appearing to cause more problems. A non-parametric Spearman's rank correlation was used in an attempt to identify those physical pipe attributes which were associated with failure events, but no conclusive relationships were established and the results were not consistent for each of the years in the data record. By far the most consistent method of predicting future sewer failure (in this case those in the last year of the data record) was to rank grid squares on the basis of total number of past events in the square, as indicated in the original paper. A more detailed discussion of the statistical analysis performed on the grid square data can be found in Sweeting. ${ }^{8}$ 